\title{
BMJ Open Alcohol consumption trajectories and self-rated health: findings from the Stockholm Public Health Cohort
}

\author{
Katalin Gémes, ${ }^{\oplus 1,2}$ Jette Moeller, ${ }^{1}$ Karin Engström, ${ }^{1}$ Anna Sidorchuk ${ }^{3}$
}

To cite: Gémes K, Moeller J, Engström K, et al. Alcohol consumption trajectories and self-rated health: findings from the Stockholm Public Health Cohort. BMJ Open 2019;9:e028878. doi:10.1136/ bmjopen-2018-028878

- Prepublication history and additional material for this paper are available online. To view please visit the journal (http:// dx.doi.org/10.1136/bmjopen2018-028878).

Received 02 January 2019 Revised 20 May 2019 Accepted 12 July 2019
Check for updates

(c) Author(s) (or their employer(s)) 2019. Re-use permitted under CC BY-NC. No commercial re-use. See rights and permissions. Published by BMJ.

${ }^{1}$ Department of Public Health Sciences, Karolinska Institutet, Stockholm, Sweden

${ }^{2}$ Institute of Environmental

Medicine, Karolinska Institutet, Stockholm, Sweden

${ }^{3}$ Center for Psychiatry Research, Department of Clinical Neuroscience, Karolinska Institute, Stockholm \& Healthcare Services, Stockholm County Council, CAP Research Center, Gävlegatan, Stockholm

Correspondence to Dr Katalin Gémes, Public Health, Karolinksa Institutet, Stockholm, Sweden; katalin.gemes@ki.se

\section{ABSTRACT}

Objective To investigate whether poor self-rated health and psychological distress are differentially associated with drinking trajectories over time.

Methods From the Stockholm Public Health Cohort, two subcohorts surveyed in 2002-2010-2014 and 2006-2010-2014 $(n=23794$ and $n=34667$ at baseline, respectively) were used. Alcohol consumption, self-rated health, psychological distress (measured by General Health Questionnaire-12), lifestyle factors and longstanding illness were assessed by questionnaires. Demographic and socioeconomic variables were obtained by register linkage. Logistic regression was fitted to assess the associations with eight alcohol consumption trajectories, which were constructed among 30228 individuals (13898 and 16330 from the 2002 and 2006 subcohorts, respectively) with measures of consumption at three time points.

Results Compared with stable moderate drinkers, all other trajectories were associated with poor self-rated health with multiadjusted $\mathrm{OR}$ for stable non-drinkers of 2.35 (95\% Cls 1.86 to 2.97), unstable non-drinkers $(\mathrm{OR}=2.58,95 \% \mathrm{Cl} 1.54$ to 3.32$)$, former drinkers $(\mathrm{OR}=2.81$, $95 \% \mathrm{Cl} 2.31$ to 3.41$)$ and stable heavy drinkers $(\mathrm{OR}=2.16$, $95 \% \mathrm{Cl} 1.47$ to 3.20$)$. The associations were not fully explained by sociodemographic and lifestyle factors and longstanding illness. Former drinking, but no other trajectories, was associated with psychological distress $(0 \mathrm{R}=1.24 ; 95 \% \mathrm{Cl} 1.10$ to 1.41$)$.

Conclusion We found a U-shape association between alcohol trajectories and self-rated health, but not with psychological distress. Compared with stable moderate drinking, former drinking was associated with the highest odds of both poor self-rated health and psychological distress. The study confirms the importance of a lifecourse approach to examining the effect of alcohol consumption on health and highlights the poorer general and mental health status of non-drinkers who were former drinkers.

\section{INTRODUCTION}

Moderate alcohol consumption is reported to be associated with lower overall mortality and a lower risk of several chronic diseases. ${ }^{12}$ Previous studies describe a so-called U-shape association showing that both non-drinkers and heavy alcohol consumers are prone to experience more adverse health outcomes, including poor subjective health and to be

\section{Strengths and limitations of this study}

The main strength of our study is the use of repeatedly measured data on alcohol intake, which allows studying the impact of over time stability and change of alcohol consumption on self-rated health and psychological distress.

- The large population-based sample with complete information on alcohol intake ensures statistical power in the analyses of distinct types of alcohol consumption trajectories.

- Comprehensive collection of health-related survey data provide a unique opportunity for extending the set of clinical outcomes of alcohol consumption to self-perceived general and mental health, which is commonly unavailable in registers and register-based research.

- The major limitation of our study is that similar to most other studies, alcohol consumption was self-reported, which might imply under-reporting of consumption, particularly among heavy drinkers.

- While we followed our cohorts for approximately 10 years, the study might still not be resistant to the 'sick-quitter' bias, as stable non-drinkers could be former heavy drinkers prior to the baseline.

at a higher risk of mental and somatic disorders. ${ }^{3-6}$ While in Sweden the total alcohol consumption is one of the lowest in Europe, ${ }^{7}$ and the average amount of alcohol consumption has been decreasing over the last decade, alcohol-related harm due to heavy consumption and hazardous drinking habits is still considerable. ${ }^{8}$ Furthermore, frequent moderate consumption of mostly wine has been getting popular, even if the evidence of health benefit of such consumption is questionable. $^{8}$

It is still not clear how long-term alcohol consumption and changes or stability of drinking habits affect general and mental health, as most previous studies rely on measuring alcohol consumption at only one point in time. ${ }^{1-5}$ As the association between alcohol consumption and health is bidirectional, current moderate alcohol consumption 
may be rather a marker of good health instead of the consequence of it, $^{9}$ and current non-drinking may instead be a consequence of a declining health. ${ }^{10}{ }^{11}$ This bidirectional association causes concerns regarding the validity of findings if alcohol consumption was assessed only at one point in time. To address these concerns, longitudinal studies with repeated measurements of both alcohol consumption and health are warranted. Studies that have examined the associations between alcohol consumption trajectories over time and adverse health outcomes also point towards a U-shape association between consumption patterns and health outcomes. For example, compared with stable moderate drinkers, individuals with a stable heavy drinking pattern and those who were former drinkers but quitted appear to have the highest risk of developing cardiovascular diseases. ${ }^{12-14} \mathrm{~A}$ higher risk of cardiovascular morbidity is also shown for long-term non-drinkers or unstable moderate drinkers compared with stable moderated drinkers, but the difference is less pronounced. ${ }^{12-14}$ Longitudinal studies on the association between alcohol consumption trajectories and quality of life and mental health reveal a similar pattern, namely that stable and unstable heavy drinkers and former drinkers have poorer quality of life and declining mental health compared with stable moderate drinkers. ${ }^{15-17}$ It is, however, less clear, whether long-term non-drinking is also associated with worsening mental health, ${ }^{15-17}$ and whether the higher risk of adverse health outcomes among long-term non-drinkers remains after taking into account other factors that could also be associated with both alcohol consumption and health, such as baseline health, socioeconomic, psychosocial and lifestyle factors. ${ }^{18}$

As previous studies have mostly focused on clinically measured health outcomes and less effort has been made to explore associations between drinking habits and self-perceived general and mental health measures that strongly relate to quality of life, this study aimed to understand whether the associations with poor self-rated health or psychological distress differ for different alcohol consumption trajectories and whether these differences are explained by sociodemographic and lifestyle factors.

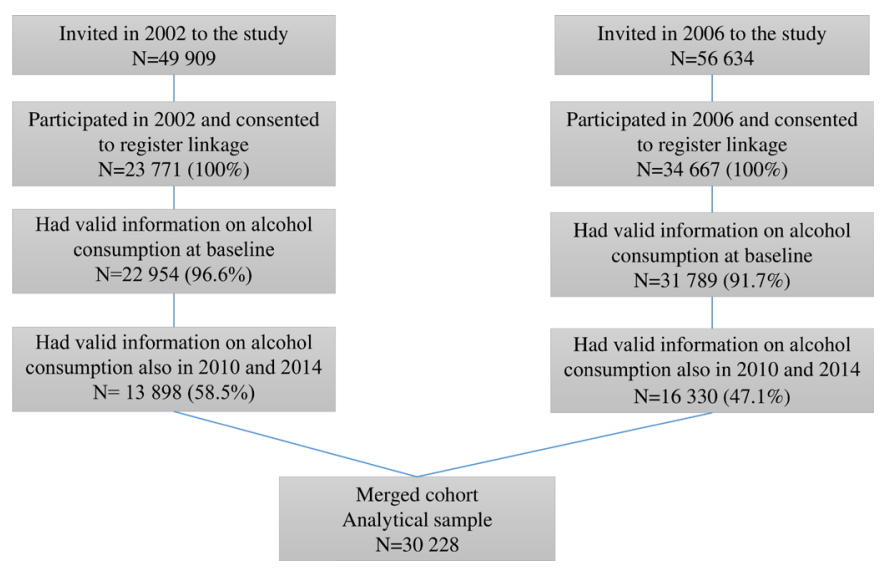

Figure 1 Flow chart of the selection of study participants.
We hypothesised that for self-rated health and psychological distress, there is a U-shape association with alcohol consumption trajectories similar to that previously reported for clinically measured somatic outcomes.

\section{METHODS}

\section{Study population}

The Stockholm Public Health Cohort (SPHC) is a population-based study that has been conducted in Sweden every fourth year since 2002, that is, in 2002, 20062007, 2010 and in 2014. In each wave, a gender and an area-specific random sample of individuals over 18 years of age and living in Stockholm County were invited and surveyed. ${ }^{19}$ Individual self-reported data were linked to administrative registers through the unique identification number that is assigned to each Swedish resident. ${ }^{20}$ Under each wave, a subcohort was created consisting of the respondents to baseline and follow-up surveys. The first subcohort was established in 2002, in which 49909 individuals were invited and 31182 completed the baseline survey. Out of respondents, 23794 (76.3\%) consented to register linkage and participated in the second survey in 2007. The third survey was conducted in $2010(\mathrm{n}=19327)$ and the fourth in $2014(\mathrm{n}=14541)$. As questions on alcohol consumption differed substantially in 2007 compared with the other surveys, in this study, we focused on information from 2002, 2010 and 2014. In the second subcohort, established in 2006, overall 56634 new individuals were invited, and 34667 have answered the baseline survey and consented to register linkage. This subcohort was followed up in $2010(\mathrm{n}=25167)$ and 2014 $(\mathrm{n}=17714)$. Individuals in both subcohorts answered the same or largely similar questions regarding their alcohol consumption habits, economic activity, lifestyle factors and somatic and mental health. Information on gender, age, socioeconomic status and marital status and country of birth were obtained from register linkage. ${ }^{21}$

\section{Study design}

In this study, data from the two subcohorts were pooled. Information on sociodemographic characteristics, lifestyle factors (i.e., alcohol consumption, smoking, physical activity and fruit consumption) and health (ie, having long-standing illness, self-rated health and psychological distress) from the first surveys (2002 and 2006, respectively) were used as the baseline measures. Self-rated health and psychological distress were assessed again in 2014. To define longitudinal alcohol trajectories, the measures of alcohol consumption at three time points, that is, at baseline and two follow-ups, within the first and the second subcohorts were used (2002-2010-2014 and 2006-20102014 , respectively). In the first subcohort, $96.6 \%$ of the participants gave valid answers on their alcohol consumption at baseline, and a total of 13898 (58.5\%) individuals reported alcohol consumption at all three measurement points (figure 1). In the second subcohort, the response rate for alcohol consumption items was $91.7 \%$ at baseline, 
and $16330(47.1 \%)$ individuals provided corresponding information in all three surveys (figure 1). Those who dropped out at any time during the study period were more likely to be non-drinkers, born outside Sweden, less likely to be employed and had more disadvantaged health behaviours and poorer self-rated health (online supplementary tables 1 and 2).

In the analytical sample, we included all individuals who gave a valid answer to the questions on alcohol consumption in all the three surveys as well as answered the question on self-rated health and/or replied to the General Health Questionnaire-12 (GHQ-12) at the baseline and the last follow-up. This yielded a final merged cohort of 30228 individuals who were followed from baseline to 2014. Thus, the members of the first subcohort with baseline survey in 2002 were followed for 12 years, and the members of the second subcohort with baseline survey in 2006 were followed for 8 years.

\section{Variables}

\section{Alcohol use}

The amount and frequency of beverage-specific alcohol consumption in centilitres were assessed for a 'typical week' with reference to 12 months prior to surveys (see online supplement appendix for details). The average weekly alcohol consumption was calculated in grams of $100 \%$ alcohol per week. ${ }^{22}$ Non-drinkers were defined as individuals who reported not having consumed alcohol in the previous year. Those who reported drinking alcohol were categorised according to their weekly alcohol consumption, using gender-specific cut-off levels. For women consuming $>0-\leq 168 \mathrm{~g}$ and for men consuming $>0-\leq 252 \mathrm{~g}$, the level of alcohol consumption was considered as light-to-moderate consumption, and these participants were referred as moderate drinkers in our study. ${ }^{53}$ Above this limit, the participants were considered as heavy drinkers (consumption of $>168 \mathrm{~g}$ for women and $>252 \mathrm{~g}$ for men). In Sweden, one standard drink contains approximately $12 \mathrm{~g}$ of $100 \%$ alcohol, ${ }^{7}$ therefore $168 \mathrm{~g}$ and $252 \mathrm{~g} 100 \%$ alcohol consumption per week refer to an average of 14 and 21 standard drinks, respectively. Based on the levels of alcohol consumption reported in three surveys, the following eight trajectory groups were defined ${ }^{11}$ (online supplementary table 3 ): (1) stable non-drinkers, (2) stable moderate drinkers and (3) stable heavy drinkers were defined as those who reported the same alcohol consumption level at all three measurement points. Individuals who reported the same alcohol consumption level at two out of three measurement points were categorised as (4) unstable non-drinkers, (5) unstable moderate drinkers and (6) unstable heavy drinkers, based on the most frequent alcohol intake level. Furthermore, (7) former drinkers were defined as those who reported non-drinking at the last measurement point and any drinking at the prior measurements. Any other types of trajectories were defined as (8) mixed.

\section{Outcomes}

Self-rated general health was measured with the standardised self-rated health question ${ }^{24}$ : 'How do you assess your overall health status?', which contains possible answers on a 5-step Likert scale. We dichotomised self-rated health to poor versus good using the answers such as 'bad' and 'very bad' as an indication of poor health and the answers as 'very good', 'good' and 'average' as an indication of being in good health. Information on self-rated health was retrieved from the baseline survey to be used as a covariate for adjustment and stratification and from the last follow-up survey in 2014 to present an outcome measure. There were 234 participants in the analytical sample who did not answer to the self-rated health question in 2014 and thus were excluded from the analysis of the corresponding outcome.

Psychological distress was measured by the GHQ-12, which is a 12-item questionnaire tool to assess symptoms of common mental disorders in population studies. SPHC employed the Swedish version translated by Diderichsen and Janlert. ${ }^{25}$ It contains six positively phrased and six negatively phrased questions on symptoms and functioning in the past few weeks with four possible answers in relation to: ability to concentrate, having lost sleep over worries, playing a useful part in society, capability of making decisions, being constantly under strain, being unable to overcome difficulties, enjoying normal day-to-day activities, facing up to problems, feeling unhappy and depressed, losing confidence in yourself, thinking of yourself as worthless person and feeling reasonably happy. Each question was provided with four response options, which for positively phrased questions included 'better (or more) than usual', 'as usual', 'worse (or less) than usual' and 'much worse (or much less) than usual', and for negatively phrased questions included 'not at all', 'not more than usual', 'more than usual' and 'much more than usual' ${ }^{25}$ We used the standard GHQ scoring (0-0-1-1) where high scores indicate distress. In line with prior study on the SPHC's GHQ data, ${ }^{26}$ a summary score was computed for the participants who answered at least 8 out of 12 items with internal missing counted as 0 . There were 185 individuals in the analytical sample who had no valid GHQ-12 information in 2014 and therefore were excluded from the analysis of psychological distress. The summary points were further categorised as no psychological distress if scoring $<4$ and as psychological distress if scoring $\geq 4$, based on a cut-off validated for the Swedish population as a measure of common mental disorders (sensitivity $=81.7$ and specificity $=85.4) .{ }^{26}$ Same as for self-rated health, baseline information on psychological distress was used for adjustment, and the follow-up GHQ-12, assessed in 2014, defined the outcome.

\section{Covariates}

Gender was categorised as men or women and age at baseline was divided into three categories: $<30$ years, $\geq 30-<49$ years and $\geq 50$ years. Country of birth was categorised as being born in or outside Sweden. Marital status 
was defined as married/cohabiting or single/divorced/ widowed. Current main daily activity was defined as employed, unemployed and others (eg, student, retired, parental leave and so on). Socioeconomic position (SEP) was based on current or past occupation and was categorised as high-skilled non-manual, middle-skilled or lower skilled non-manual, manual workers and self-employed, according to the Swedish socioeconomical classification. ${ }^{27}$ Participants were considered physically active if they reported at least three times of 30 min moderate activity per week or, otherwise, inactive. ${ }^{28}$ As tobacco use was assessed with slightly different questions in 2002 and 2006, the following categories were used to capture the same information in both subcohorts: daily tobacco users (ie, daily smokers/smokeless tobacco users) and non-daily tobacco users (ie, occasional, former or non-smokers/ smokeless tobacco users). The frequency of fruit consumption was used as a proxy for diet and was categorised as high fruit consumption, if reported eating fruits/berries at least once a day or, otherwise, as low fruit consumption. ${ }^{29}$ Having long-standing illnesses was defined as yes or no, based on the answers to the question of 'Do you suffer from a long-term illness, health problems following an accident, disability or other persistent health problems?'.

\section{Statistical analysis}

$\chi^{2}$ test and analysis of variance were conducted to compare the distribution of study variables at baseline among individuals with different alcohol consumption trajectories and, separately, between the subcohorts. Logistic regression was used to assess the association between alcohol trajectory group membership and self-rated health and psychological distress measured in 2014. The first model was minimally adjusted for the subcohort membership and the baseline values of the outcomes. In the multiadjusted analysis, age, gender, SEP, country of birth, current main activity, lifestyle factors (ie, tobacco use, physical activity and fruit consumption) and long-standing illnesses were added to the model, as they were previously shown to influence the association between alcohol consumption and health. We excluded the mixed trajectory group from further analysis due to the low number of participants in the group.

Average weekly alcohol consumption in the study waves according to alcohol trajectories

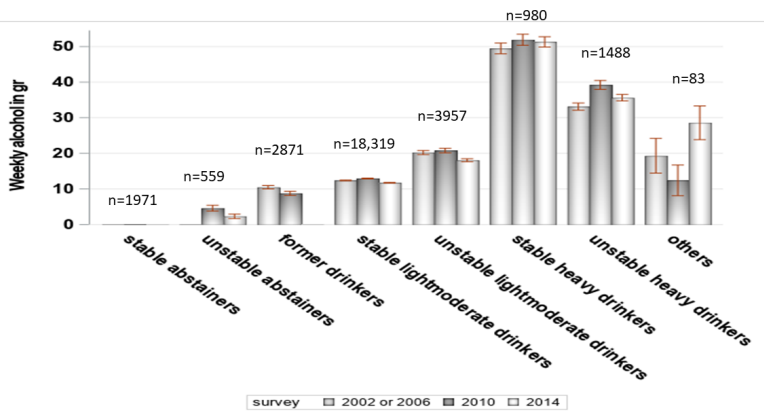

Figure 2 Average alcohol consumption in the study waves according to alcohol consumption trajectories.
In the sensitivity analysis, we stratified the main analysis by baseline outcome status, gender, age, subcohort membership and having long-standing illnesses to examine whether the associations are modified by these covariates. We also examined whether baseline poor self-rated health and psychological distress predict the membership in the different alcohol consumption trajectories. To explore the association between alcohol consumption trajectories and changes in self-rated health and psychological distress from baseline to 2014, we created two variables: (1) change in self-rated health and (2) change in psychological distress. Both variables were categorised as decreasing, increasing and no change. For self-rated health, any differences in the Likert scale between the baseline and 2014 values were considered as a change, while for psychological distress, two-point difference between the baseline and 2014 measures on the GHQ-12 scale was considered as a change. We conducted multinomial regression analysis using the change in selfrated health and psychological stress as outcomes. Finally, we applied multinomial logistic regression to examine whether self-rated health categorised as 'very good/ good', 'average', 'poor/very poor' would lead to different results compared with the dichotomised outcome ('poor' vs 'good').

To handle missing values on covariates, we used a complete case analysis, but we also compared the distribution of baseline alcohol consumption, self-rated health and GHQ scores among those who did not answer the follow-up questionnaires and those who answered all three questionnaires.

\section{Patient and public involvements}

No patients or public were involved in the development of the research questions, the design and implementation of the study or the outcome measure. The study results will be communicated to healthcare professionals via scientific conferences and disseminated to the general public through the Stockholm County Council Health Stockholm website (http://folkhalsoguiden.se/halsastockholm/published-studies/).

\section{RESULTS}

The distribution of baseline characteristics was similar in both subcohorts, except for employment status and tobacco use, which differed due to a slight change in the corresponding questions between 2002 and 2006 (online supplementary table 4 , figure 2 ).

Table 1 shows the distribution of the baseline characteristics in the merged cohort according to the alcohol consumption trajectory groups. The majority of the participants followed stable or unstable moderated alcohol consumption trajectories $(61 \%$ and $13 \%$, respectively). Almost $10 \%$ of the individuals were former drinkers. The proportions of women, non-Swedish born, high-skilled non-manual workers and those with longstanding illness were highest among stable and unstable non-drinkers. 


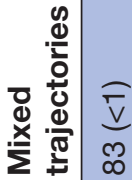

䍃总站

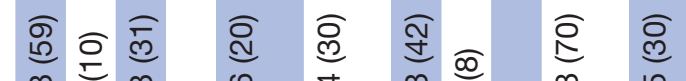

ब्ले $\bar{\epsilon}$

ले โ

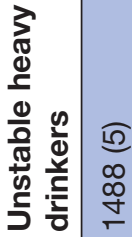

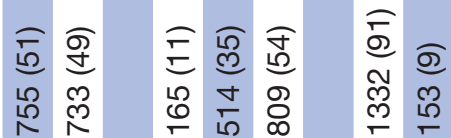

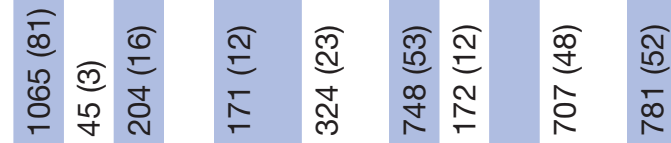

命䞡

กิ๊ $\bar{\varnothing}$

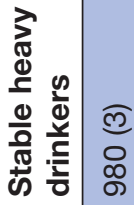

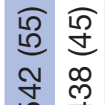

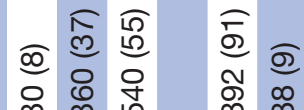

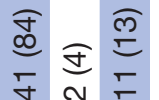

또ำ

苞

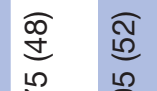

可通

ㅇำ

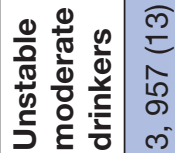

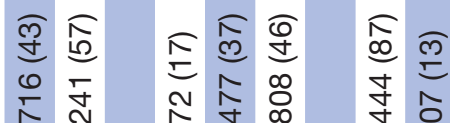

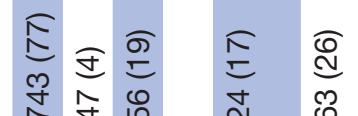

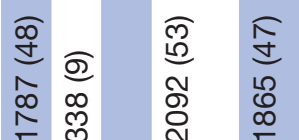

$\stackrel{\infty}{\sim} \stackrel{p}{\Sigma}$

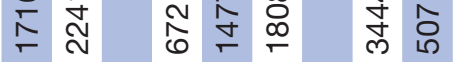

竎守

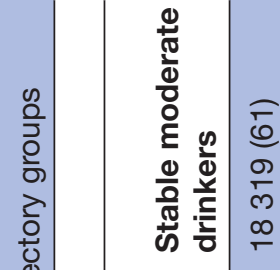

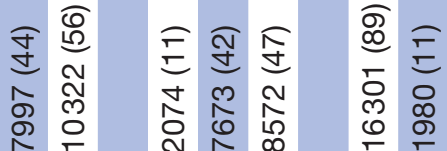

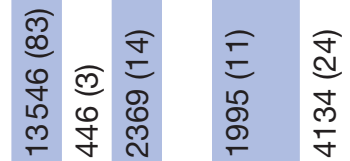

苞

ֻू

合

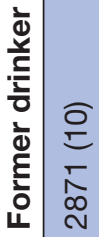

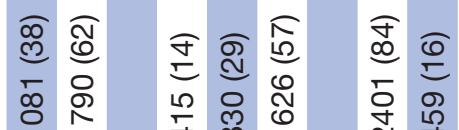

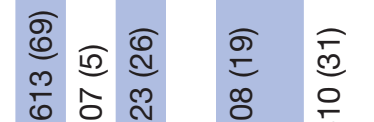

尔

赑

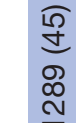

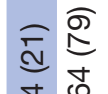

空

$\stackrel{m}{\frac{2}{2}}$

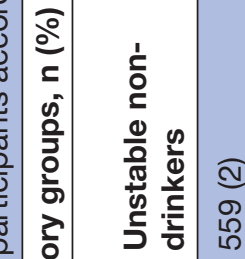

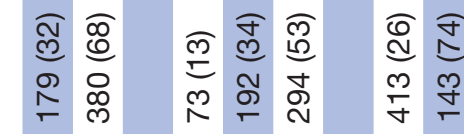

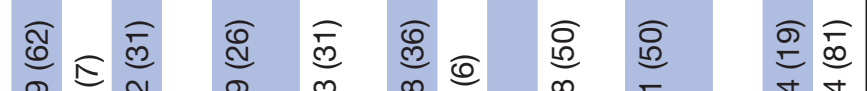

总

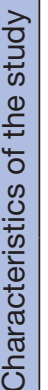

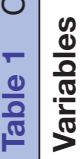

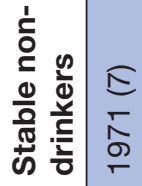

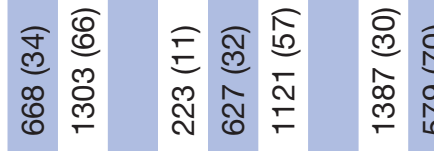

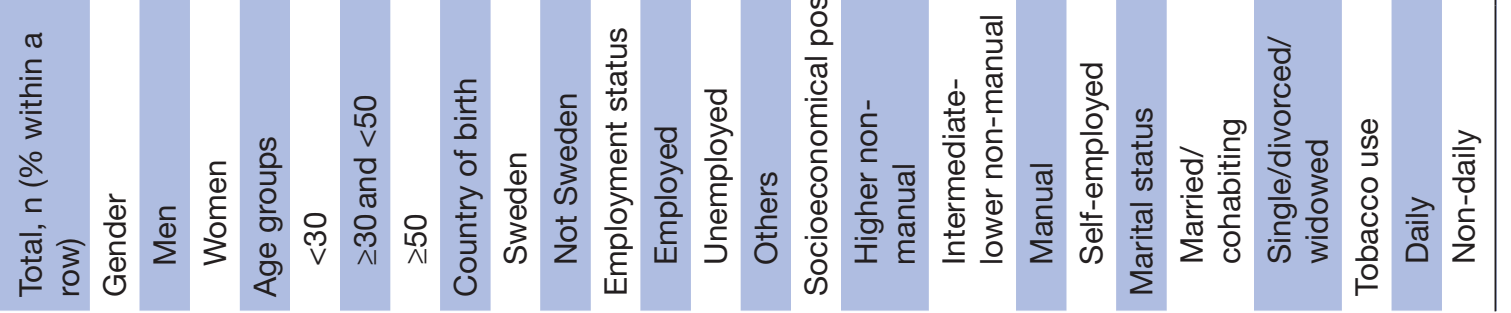




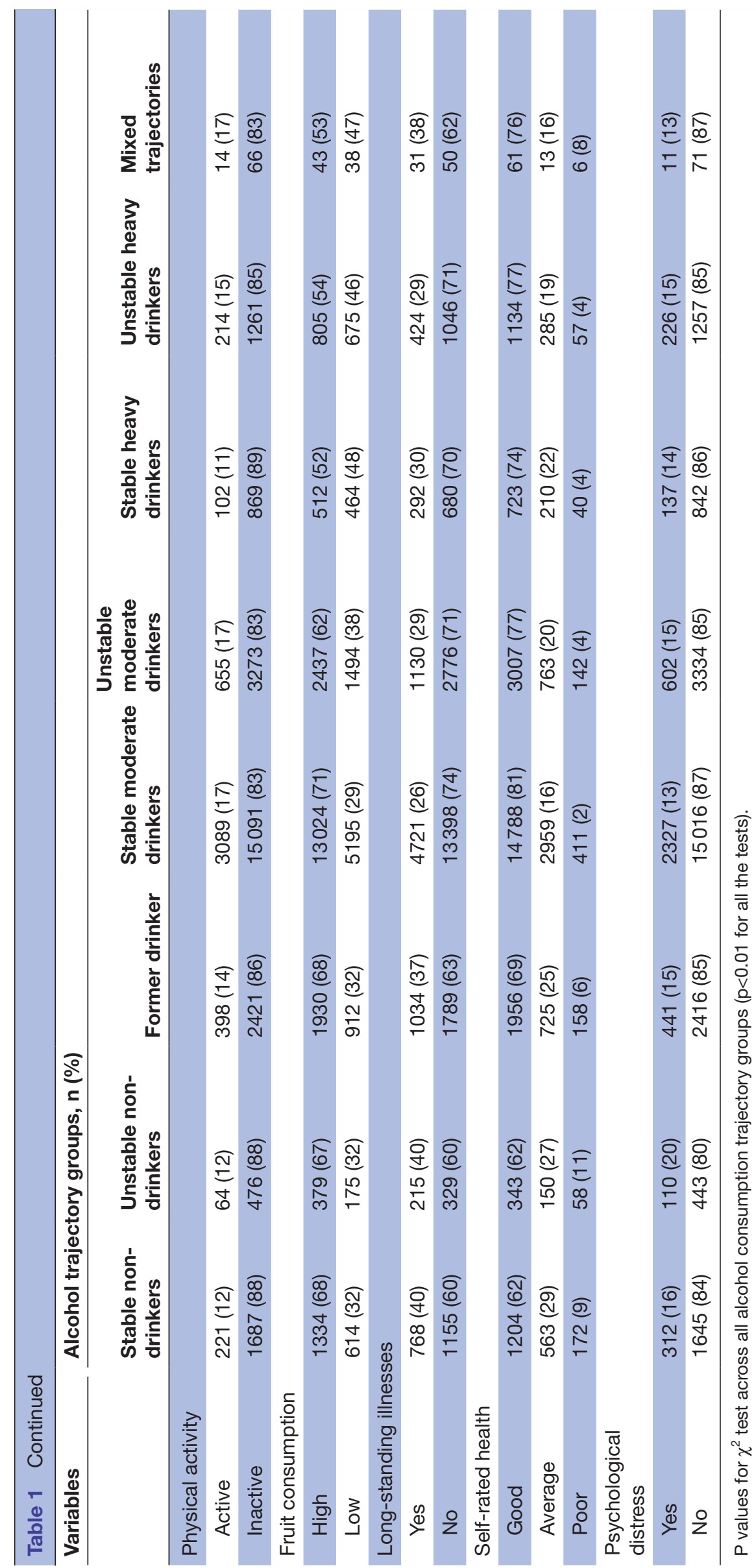

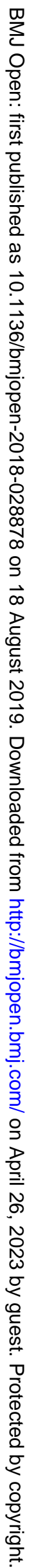


Table 2 Association between alcohol consumption trajectory group membership and poor self-rated health and psychologica distress in 2014

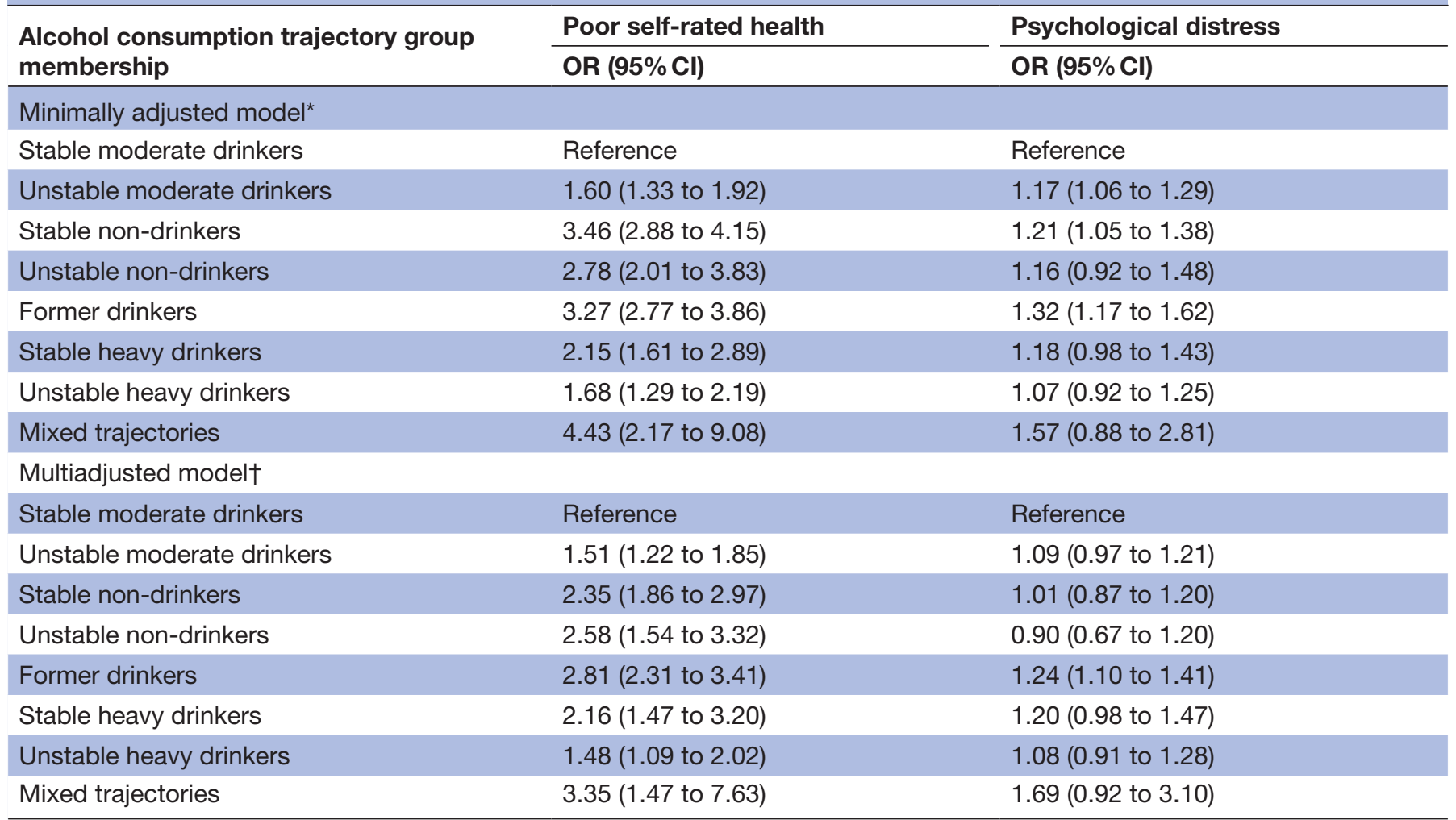

${ }^{*}$ Adjusted for cohort membership and baseline status of the outcome.

†Additionally adjusted for age, gender, marital status, country of birth, current main activity, socioeconomic position, tobacco use, physical activity, fruit consumption and long-standing illness. Logistic regression was used to analyse the association between alcohol consumption trajectories and poor self-rated health and psychological distress. Self-rated health was measured in 2014 and dichotomised as '(very) poor' and '(very) good average'. Psychological distress was measured in 2014 and defined as a score of $\geq 4$ according to the General Health Questionnaire-12. The OR for the association between baseline measure of self-rated health and the corresponding measure in 2014 was 5.73 (95\% Cl 4.74 to 6.94). Likewise, the association between baseline measure of psychological stress and the corresponding measure in 2014 was 4.00 (95\% Cl 3.71 to 4.32$)$.

While the proportions of men, Swedish born, self-employed, daily tobacco users and those who reported low fruit consumption were highest in stable and unstable heavy drinkers.

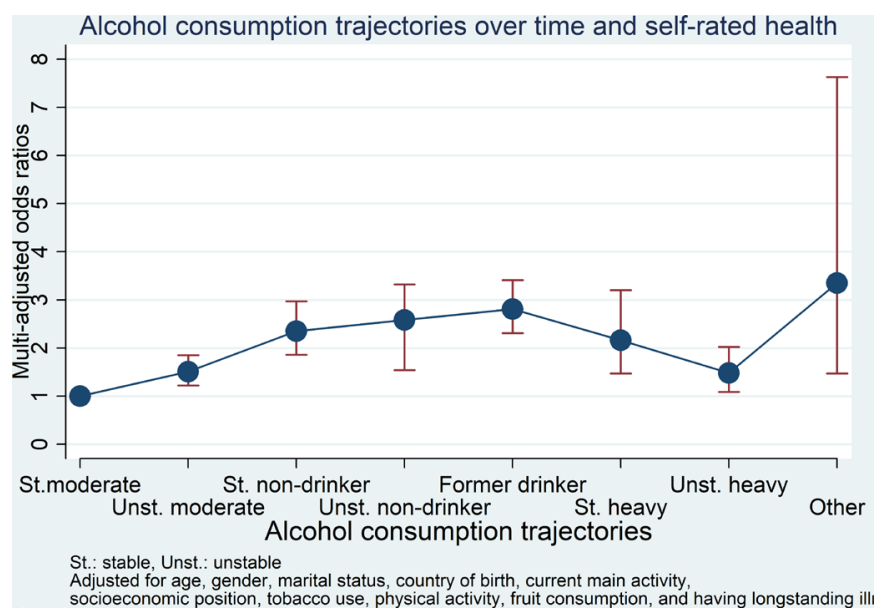

Figure 3 Alcohol consumption trajectories and poor selfrated health.
The associations of alcohol consumption trajectories with self-rated health and psychological distress are shown in table 2, figures 3 and 4. Compared with stable moderate drinkers, an elevated likelihood of having poor self-rated health appeared among the members of all other trajectories, regardless of the subcohort membership, baseline outcome values and adjustment for other covariates with an estimated OR range between 1.2 and 4.4. For psychological distress, a minimally adjusted model revealed an association with unstable moderate drinking, stable non-drinking, former drinking and mixed trajectories, while further adjustment for sociodemographic and lifestyle covariates and long-standing illnesses eliminated most of the associations, apart from that in former drinkers (OR of $1.24,95 \%$ CIs 1.10 to 1.41 ).

\section{Sensitivity analyses}

Stratification by the baseline value of self-rated health did not alter the results considerably (table 3). In 25853 individuals with no psychological distress at baseline, an association with a high score for distress in 2014 was observed for former drinkers. No significant associations 
Alcohol consumption trajectories over time and psychological distress

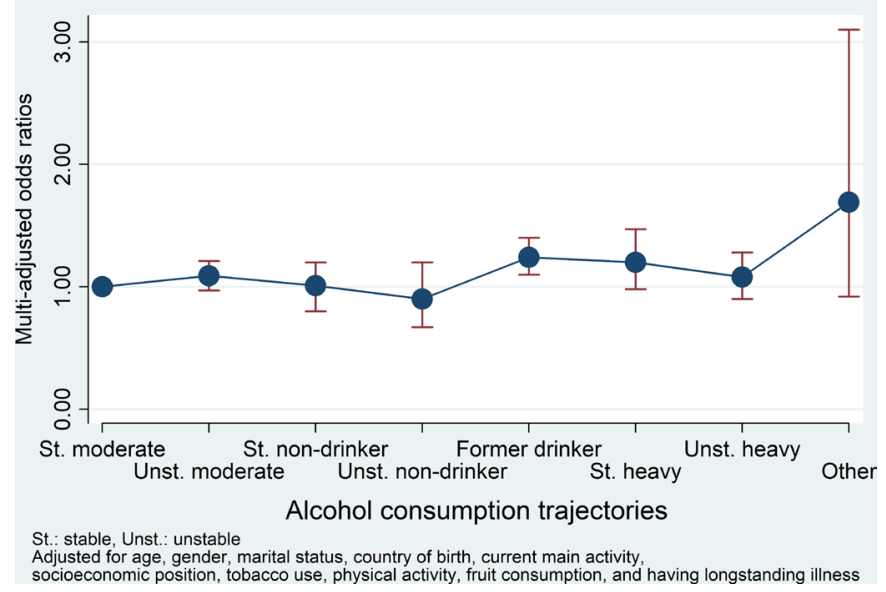

Figure 4 Alcohol consumption trajectories and psychological distress.

with psychological distress at the end of follow-up was observed in 4155 individuals who scored high for distress at baseline. The odds being a member of trajectories such as stable or unstable non-drinkers, heavy drinkers and former drinkers were higher for those with poor selfrated health at baseline (online supplementary table 5).
Psychological distress at baseline increased the odds of being a member of unstable heavy drinking trajectories and unstable non-drinking trajectories (online supplementary table 5). Examining the association between alcohol trajectories and change in self-rated health and psychological distress showed that former drinkers and stable heavy drinkers had higher odds of worsening selfrated and mental health (online supplementary table 6). Stable non-drinkers only showed a worsening self-rated health but not worsening mental health . For other trajectories, no association with change in self-rated health or psychological stress appeared. Stratification by gender revealed no major differences in the likelihood of having poor self-rated health and psychological distress between men and women (table 4). Stratification by the subcohort membership revealed slightly stronger associations with poor self-rated health among members of the second subcohort with no clear difference between the subcohorts regarding psychological distress (online supplementary table 7). No major differences were seen in the analysis stratified by age at baseline (online supplementary table 8). Among individuals with long-standing illness at baseline, unstable moderate drinking, former drinking and stable heavy drinking were associated with a slightly

Table 3 Association between alcohol consumption trajectory group membership and self-rated general health and psychological distress reported in 2014 , stratified by baseline outcome values

\begin{tabular}{|c|c|c|c|}
\hline \multirow[b]{3}{*}{$\begin{array}{l}\text { Alcohol consumption } \\
\text { trajectory group membership }\end{array}$} & OR $(95 \% \mathrm{Cl})$ & OR $(95 \% \mathrm{Cl})$ & P for interaction \\
\hline & \multicolumn{3}{|l|}{ Self-rated general health } \\
\hline & $\begin{array}{l}\text { Good-average at baseline } \\
(n=28810)\end{array}$ & $\begin{array}{l}\text { Poor at baseline } \\
(n=1038)\end{array}$ & \\
\hline Stable moderate drinkers & Reference & Reference & \\
\hline Unstable moderate drinkers & 1.44 (1.14 to 1.82$)$ & 1.18 (0.72 to 1.92$)$ & 0.844 \\
\hline Former drinkers & 3.08 (2.49 to 3.80$)$ & 3.54 (1.73 to 7.24$)$ & 0.030 \\
\hline Stable heavy drinkers & 2.20 (1.54 to 3.13$)$ & $1.44(0.65$ to 3.21$)$ & 0.518 \\
\hline \multirow[t]{2}{*}{ Unstable heavy drinkers } & 1.42 (1.00 to 2.03$)$ & $1.59(0.82$ to 3.11$)$ & 0.580 \\
\hline & \multicolumn{3}{|l|}{ Psychological distress (PS) } \\
\hline Unstable moderate drinkers & 1.11 (0.97 to 1.26$)$ & $1.04(0.84$ to 1.29$)$ & 0.564 \\
\hline Stable non-drinkers & $0.93(0.76$ to 1.13$)$ & $1.22(0.91$ to 1.64$)$ & 0.072 \\
\hline Unstable non-drinkers & 0.85 (0.59 to 1.23$)$ & 0.93 (0.57 to 1.52$)$ & 0.613 \\
\hline Former drinkers & 1.29 (1.11 to 1.49$)$ & $1.12(0.89$ to 1.43$)$ & 0.316 \\
\hline Stable heavy drinkers & 1.16 (0.91 to 1.49$)$ & 1.38 (0.93 to 2.05$)$ & 0.356 \\
\hline Unstable heavy drinkers & 1.04 (0.84 to 1.29$)$ & $1.19(0.87$ to 1.63$)$ & 0.373 \\
\hline
\end{tabular}

Psychological distress was measured by the General Health Questionnaire-12. All analyses were adjusted for age, gender, marital status, country of birth, current main activity, socioeconomic position, tobacco use, physical activity, fruit consumption and longstanding illness. Logistic regression was used to analyse the association between alcohol consumption trajectories and poor self-rated health and psychological distress reported 2014. Self-rated health was dichotomised as '(very)poor' and '(very) good, average'. Psychological distress was defined as a score of $\geq 4$ on the General Health Questionnaire-12. 
Table 4 Association between alcohol trajectory memberships and self-rated health and psychological distress in 2014, stratified by gender

\begin{tabular}{|c|c|c|c|}
\hline & OR $(95 \% \mathrm{Cl})$ & OR $(95 \% \mathrm{Cl})$ & \multirow{2}{*}{$P$ for interaction } \\
\hline & $\begin{array}{l}\text { Women } \\
(n=12977)\end{array}$ & $\begin{array}{l}\text { Men } \\
(n=17 \text { 251) }\end{array}$ & \\
\hline Stable moderate drinkers & Reference & Reference & \\
\hline Unstable moderate drinkers & 1.33 (0.94 to 1.87$)$ & $1.48(1.13$ to 1.94$)$ & 0.464 \\
\hline Former drinkers & 3.24 (2.37 to 4.42 ) & 2.55 (1.98 to 3.27$)$ & 0.264 \\
\hline Stable heavy drinkers & 1.78 (1.10 to 2.87$)$ & 2.39 (1.53 to 3.75$)$ & 0.240 \\
\hline \multirow[t]{2}{*}{ Unstable heavy drinkers } & 1.65 (1.07 to 2.55$)$ & 1.33 (0.85 to 2.07 ) & 0.611 \\
\hline & \multicolumn{2}{|c|}{ Psychological distress (PS) } & \\
\hline Unstable non-drinkers & 1.15 (0.67 to 1.97$)$ & $0.82(0.58$ to 1.16$)$ & 0.300 \\
\hline Former drinkers & 1.37 (1.09 to 1.72$)$ & 1.19 (1.02 to 1.38$)$ & 0.330 \\
\hline Stable heavy drinkers & 1.20 (0.88 to 1.63$)$ & 0.82 (0.58 to 1.16$)$ & 0.836 \\
\hline Unstable heavy drinkers & $1.07(0.81$ to 1.41$)$ & 1.10 (0.88 to 1.38$)$ & 0.810 \\
\hline
\end{tabular}

Psychological distress was measured by the General Health Questionnaire-12. All analyses were adjusted for age, gender, marital status, country of birth, current main activity, socioeconomic position, tobacco use, physical activity, fruit consumption and long-standing illness. Logistic regression was used to analyse the association between alcohol consumption trajectories and poor self-rated health and psychological distress measured in 2014. Self-rated health was dichotomised as '(very) poor' and '(very) good, average'. Psychological distress was defined as a score of $\geq 4$ on the General Health Questionnaire-12.

higher likelihood of having psychological distress than in individuals without such illnesses (online supplementary table 9). Analysis of self-rated health categorised as good/ very good, average and poor/very poor provided essentially the same results as for the dichotomised outcome (online supplementary table 10).

\section{DISCUSSION}

We observed a U-shape association between alcohol consumption trajectories and self-rated health. Individuals with stable moderate consumption trajectory appeared to have a better self-rated health than the members of all other trajectories and the associations were not fully explained by demographic, socioeconomic and lifestyle factors, and long-standing illness. Concerning psychological distress, the associations were weaker and diminished when study covariates were controlled for. Only former drinkers appeared to have a higher likelihood of psychological distress compared with stable moderate drinkers regardless of the adjustment strategy. Overall, the associations did not differ between genders and age groups and were stronger in individuals with long-standing illness at baseline.

\section{Comparison with previous studies}

Our results were in line with other studies, which suggested a U-shape association between alcohol consumption over time and adverse self-reported and clinically measured health outcomes. ${ }^{18} 3031$ An Australian study on self-rated quality of life in middle-aged women reported long-term moderate drinkers to have the best self-reported general health compared with any other drinking trajectories, and any changes in moderate alcohol consumption were associated with a decline in general health. ${ }^{18}$ The U-shape association was also observed in relation to cardiovascular ill-health as long-term moderate drinkers were reported to have less pronounced arterial ageing, ${ }^{11}$ the lowest level of inflammatory cytokines ${ }^{17}{ }^{32}$ and the lowest incidence of ischaemic heart diseases. ${ }^{13}$ Concerning mental health, long-term moderate drinkers were reported to be at the lowest risk of dementia ${ }^{15}$ and common mental disorders ${ }^{14}$ when compared with individuals with other drinking patterns (ie, former-drinkers, long-term non-drinkers or heavy drinkers). In our study, the associations between alcohol consumption trajectories and psychological distress were less clear than that for self-rated health and were mainly explained by sociodemographic and lifestyle factors as well as by long-standing illnesses. Former drinking, however, appeared to be strongly related to 
psychological distress independent of any study covariates. It is worth mentioning that former drinkers may involve individuals who quitted drinking due to worsening health or chronic medical conditions, which itself can negatively affect mental health. Likewise, former heavy drinkers who quitted alcohol consumption may also be at higher risk of mental health problems. ${ }^{33}$

Our results showed a slightly higher likelihood of having poor self-rated health among unstable moderate drinkers compared with stable moderate drinkers, which is in line with previous research where both increase and decrease in alcohol consumption among moderate drinkers were associated with deterioration of health. ${ }^{34}$ Others found that stable moderate drinking was associated with slower arterial ageing and lower risk of dementia compared with unstable moderate drinking. ${ }^{12}{ }^{15}$ In our study, the difference between stable and unstable moderate drinkers was only seen in relation to self-rated health. An elevated likelihood of psychological distress among unstable moderate drinkers compared with stable moderate drinkers, which was observed in the unadjusted analysis, appeared to be explained by psychosocial, socioeconomic and lifestyle factors, and long-standing illness, which is partly in line with previous findings where harmful alcohol use and poor general and mental health were suggested to follow a social gradient. ${ }^{35} 36$

An elevated likelihood of poor self-rated health among stable non-drinkers corresponds to the findings from other studies that report an impaired health among non-drinkers already in young adulthood. ${ }^{87}$ While in Sweden, due to strict alcohol policy, the prevalence of non-occasional and occasional drinking is higher than that in the UK or the USA, it seems that long-term non-drinkers are still a specific subgroup in this population with worse general and mental health. ${ }^{38-41}$

\section{Limitations}

There are some limitations to our study that should be considered. First, even if we followed our cohorts for approximately 10 years, our study might not be immune to the 'sick-quitter' bias, ${ }^{8}$ as stable non-drinkers could be former heavy drinkers prior to the baseline. It is, however, worth mentioning that we found similarity in elevated likelihoods of poor health among non-drinkers in the age-stratified analysis. Second, the influence of non-participation in the SPHC should be considered. ${ }^{18}$ Those who were invited to each wave but never responded to invitation were more likely men, younger than 45 years, born outside Sweden and with lower education and income than responders, ${ }^{19}$ which collectively may limit generalisability. Third, heavy drinkers might be undersampled in population-based studies, therefore caution is needed to draw inference for this group. ${ }^{10.38}$ Furthermore, some selection bias may have occurred due to loss to follow-up as heavy drinkers, non-drinkers and participants with worse baseline health represented the highest proportion of those who dropped-out in our study (online supplementary table 1 and 2). This may result in lower observed estimates among heavy drinkers and non-drinkers trajectories in our analytical sample than if drop outs would have occurred in random. Fourth, alcohol consumption was self-assessed, therefore could be under-reported, especially among heavy drinkers. As a result, we could underestimate the proportion of real heavy alcohol consumers and the proportion of stable high-drinkers and could overestimate the percentage of moderate alcohol consumers and the proportion of stable moderate drinkers in the population. However, as moderate alcohol consumers had the lowest risk of poor health, a possible misclassification of some heavy drinkers to moderate drinkers, if present, would slightly underestimate the risk of poor health among heavy drinkers. Finally, SPHC as other population surveys exclude some marginalised groups as homeless and institutionalised, which limits generalisability.

\section{CONCLUSIONS}

We found evidence of the U-shape association between longterm alcohol consumption and self-rated health, but with a less clear shape in relation to psychological distress. While stable moderate drinking was associated with the lowest odds of poor self-rated health, former drinking was associated with both the highest likelihood of poor self-rated health and psychological distress compared with moderate alcohol consumption. Our study supports the importance of longitudinal measures and life course approach in examining the effect of alcohol consumption on health and highlights the poorer self-rated general and mental health of those non-drinkers who are former drinkers.

Contributors All authors conceptualised and designed the study and the empirical analysis. AS and KG acquired data, conceptualised the variables and provided data management. $\mathrm{KG}$ carried out the statistical analysis and drafted the initial manuscript. All authors interpreted the results and critically reviewed the manuscript. All authors contributed to revising and editing the manuscript with substantial methodological and intellectual support and approved the manuscript as submitted.

Funding This study was financially supported through research grant from the European Foundation for Alcohol Research (EA 16-45 to JM), a joint grant of the Swedish Council for Information on Alcohol and Other Drugs (CAN) the Swedish Alcohol Retailing Monopoly (Systembolagets Alkoholforskningsråd) (2016-0097 to AS) and a grant from the Dutch Beer Institute (2018 to KG).

Competing interests None declared.

Patient consent for publication Obtained.

Provenance and peer review Not commissioned; externally peer reviewed.

Data availability statement Data may be obtained from a third party and are not publicly available.

Open access This is an open access article distributed in accordance with the Creative Commons Attribution Non Commercial (CC BY-NC 4.0) license, which permits others to distribute, remix, adapt, build upon this work non-commercially, and license their derivative works on different terms, provided the original work is properly cited, appropriate credit is given, any changes made indicated, and the use is non-commercial. See: http://creativecommons.org/licenses/by-nc/4.0/.

\section{REFERENCES}

1. Klatsky AL. Alcohol and cardiovascular diseases: where do we stand today? J Intern Med 2015;278:238-50.

2. Poli A, Marangoni F, Avogaro A, et al. Moderate alcohol use and health: a consensus document. Nutr Metab Cardiovasc Dis 2013;23:487-504. 
3. Boden JM, Fergusson DM. Alcohol and depression. Addiction 2011;106:906-14.

4. Stranges S, Notaro J, Freudenheim JL, et al. Alcohol drinking pattern and subjective health in a population-based study. Addiction 2006;101:1265-76.

5. Roerecke M, Rehm J. Alcohol intake revisited: risks and benefits. Curr Atheroscler Rep 2012;14:556-62.

6. Kellerborg K, Danielsson A-K, Allebeck P, et al. Disease burden attributed to alcohol: how methodological advances in the global burden of disease 2013 study have changed the estimates in Sweden. Scand J Public Health 2016;44:604-10.

7. Word Health Organization. Global status report on alcohol and health 2018. Geneva.

8. Trolldal B, Leifman H. Hur mycket dricker svensken? In: Registrerad och oregistrerad alkoholkonsumtion 2001-2014. can Rapport 152, can. Stockholm: Centralförbundet för alkohol- och narkotikaupplysning, 2015.

9. Ng Fat L, Cable N, Marmot MG, et al. Persistent long-standing illness and non-drinking over time, implications for the use of lifetime abstainers as a control group. J Epidemiol Community Health 2014;68:71-7.

10. Shaper AG, Wannamethee G, Walker M. Alcohol and mortality in British men: explaining the U-shaped curve. The Lancet 1988;332:1267-73.

11. Britton A, Ben-Shlomo Y, Benzeval M, et al. Life course trajectories of alcohol consumption in the United Kingdom using longitudinal data from nine cohort studies. BMC Med 2015;13:47.

12. Britton A, Hardy R, Kuh D, et al. Twenty-Year trajectories of alcohol consumption during midlife and atherosclerotic thickening in early old age: findings from two British population cohort studies. BMC Med 2016;14:111.

13. O'Neill D, Britton A, Brunner EJ, et al. Twenty-Five-Year alcohol consumption trajectories and their association with arterial aging: a prospective cohort study. J Am Heart Assoc 2017;6.

14. O'Neill D, Britton A, Hannah MK, et al. Association of longitudinal alcohol consumption trajectories with coronary heart disease: a meta-analysis of six cohort studies using individual participant data. BMC Med 2018;16:124.

15. Goodwin L, Norton S, Fear NT, et al. Trajectories of alcohol use in the UK military and associations with mental health. Addict Behav 2017;75:130-7.

16. Sabia S, Fayosse A, Dumurgier J, et al. Alcohol consumption and risk of dementia: 23 year follow-up of Whitehall II cohort study. BMJ 2018;362.

17. Bell S, Britton A. Drinking pattern during midlife and risk of developing depression during 28 years of follow-up: a prospective cohort study. Drug Alcohol Depend 2015;155:111-7.

18. Powers JR, Young AF. Longitudinal analysis of alcohol consumption and health of middle-aged women in Australia. Addiction 2008;103:424-32.

19. Svensson AC, Fredlund P, Laflamme L, et al. Cohort profile: the Stockholm public health cohort. Int J Epidemiol 2013;42:1263-72.

20. Ludvigsson JF, Otterblad-Olausson P, Pettersson BU, et al. The Swedish personal identity number: possibilities and pitfalls in healthcare and medical research. Eur J Epidemiol 2009;24:659-67.

21. Integrated database for labour market research: statistics Sweden 2009.

22. Andréasson $S$, Allebeck $P$, Romelsjö A. Alcohol and mortality among young men: longitudinal study of Swedish conscripts. Br Med J 1988;296:1021-5.

23. Shield KD, Gmel G, Gmel G, et al. Life-Time risk of mortality due to different levels of alcohol consumption in seven European countries: implications for low-risk drinking guidelines. Addiction 2017:112:1535-44.

24. Bjorner J, Sondergaard Kristensen T, Orth-Gomér K, et al. SelfRated health. A useful concept in research, prevention and clinical medicine. Stockholm: Swedish council for planning and coordination of research, 1996.

25. Diderichsen $F$, Janlert $U$. Effects of economic change on male morbidity in neighbouring industrial and rural municipalities in northern Sweden. J Epidemiol Community Health 1992;46:605-7.

26. Lundin A, Åhs J, Åsbring N, et al. Discriminant validity of the 12-Item version of the general health questionnaire in a Swedish case-control study. Nord J Psychiatry 2017;71:171-9.

27. . Reports on statistical co-ordination; 1982.

28. World Health Organization. Global recommendations on physical activity for health, 2010.

29. World Health Organization. Healthydiet fact sheet No. 394, 2015.

30. Schrieks IC, Wei MY, Rimm EB, et al. Bidirectional associations between alcohol consumption and health-related quality of life amongst young and middle-aged women. $J$ Intern Med 2016;279:376-87.

31. Gonzalez-Rubio E, Mauro S I, Lopez-Ruiz C, et al. Relationship of moderate alcohol intake and type of beverage with health behaviors and quality of life in elderly subjects. Qual Life Res 2016.

32. Bell S, Mehta G, Moore K, et al. Ten-Year alcohol consumption typologies and trajectories of C-reactive protein, interleukin- 6 and interleukin-1 receptor antagonist over the following 12 years: a prospective cohort study. J Intern Med 2017;281:75-85.

33. Sarich $\mathrm{P}$, Canfell K, Banks E, et al. A prospective study of health conditions related to alcohol consumption cessation among 97,852 drinkers aged 45 and over in Australia. Alcohol Clin Exp Res 2019;43:710-21.

34. Bell S, Britton A. An exploration of the dynamic longitudinal relationship between mental health and alcohol consumption: a prospective cohort study. BMC Med 2014;12:91.

35. Closing the gap in a generation: health equity through action on the social determinants of health. Final report of the Commission on social determinants of health; 2008.

36. Bloomfield K, Grittner U, Kramer S, et al. Social inequalities in alcohol consumption and alcohol-related problems in the study countries of the EU concerted action 'Gender, Culture and Alcohol Problems: a Multi-national Study'. Alcohol Alcohol Suppl 2006;41(1):i26-36.

37. Power C, Rodgers B, Hope S. U-Shaped relation for alcohol consumption and health in early adulthood and implications for mortality. The Lancet 1998;352.

38. Dumais A, De Benedictis L, Joyal C, et al. Profiles and mental health correlates of alcohol and illicit drug use in the Canadian population: an exploration of the J-curve hypothesis. Can J Psychiatry 2013;58:344-52.

39. Britton A, O'Neill D, Bell S. Underestimating the alcohol content of a glass of wine: the implications for estimates of mortality risk. Alcohol Alcohol 2016;51:609-14.

40. Imtiaz S, Loheswaran G, Le Foll B, et al. Longitudinal alcohol consumption patterns and health-related quality of life: results from the National epidemiologic survey on alcohol and related conditions. Drug Alcohol Rev 2018;37:48-55.

41. Kaplan MS, Huguet N, Feeny D, et al. Alcohol use patterns and trajectories of health-related quality of life in middle-aged and older adults: a 14-year population-based study. J Stud Alcohol Drugs 2012;73:581-90. 